\title{
COVER ESSAY
}

\section{Mixed progress against lung cancer}

\author{
Michael J Thun
}

Although tobacco smoking causes many diseases and affects most of the organ systems in the body, lung cancer is typically the first condition that comes to mind when one considers the health impact of smoking. Lung cancer is also one of the most important and devastating illnesses caused by smoking, given its high incidence in populations where smoking is common, its high fatality rate, and the difficulty in detecting the disease when it is still localised. Therefore we commissioned an update on lung cancer and smoking from Dr Michael Thun of the American Cancer Society, which appears below.

Shown on the cover of this issue of "Tobacco Control" are four false-colour scanning electron micrographs of lung cancer, obtained from the Science Photo Library in London (http://www.sciencephoto.com). The upper left image shows cancer of the human bronchus, the most common form of lung cancer. Until the 1970s, such cancers usually occurred near the division of the trachea or large bronchi into smaller bronchi, because this area suffers from heavy deposition of the carcinogenic tars from high tar cigarettes. However, squamous carcinomas in large airways have now become less common than adenocarcinomas in small, peripheral airways, paralleling the shift from "high" to "medium" and "lower" yield cigarettes, the smoke from which is inhaled more deeply. The disorganised region of malignant tumour cells at the bottom right of this image is seen invading the normal, ciliated epithelium (or lining) of the bronchus at the left and top. Cancers consist of primitive cells which have not developed any function, such as the cilia of normal, bronchial epithelial cells. (The cilia are small, hairlike projections used to sweep mucus, debris, and microorganisms out of the lung.)

The upper right image shows a tiny lung cancer (orange) filling an alveolus (one of the blind-ended air sacs which make up the lungs). The individual cancer cells are coated with microscopic, hair-like structures called microvilli. Several cancer cells can be seen separated from the main tumour. The lower left image also shows a tiny lung tumour (red), coated with microvilli, filling an alveolus.

The lower right image shows alveoli of the human lung in a smoker with lung cancer. The thinwalled alveoli are seen containing red blood cells. The blood has haemorrhaged from the walls of the alveoli due to damage caused by lung cancer. The patient was a heavy smoker and the affected lung had to be surgically removed.

Photo credits for cover illustrations: Dr Tony Brain (upper left), Moredun Animal Health (upper right and lower left), and Eye of Science (lower right). Magnifications: $\times 180$ (upper left), $\times 445$ (upper right), $\times 1190$ (lower left), and $\times 245$ (lower right). - ED

American Cancer Society, 1599 Clifton Road, NE, Atlanta, Georgia 30329, USA. mthun@ cancer.org Michael J Thun

\section{Trends in lung cancer}

Worldwide, lung cancer has become the most common site of cancer death, claiming an estimated 945000 lives each year. ${ }^{1}$ Deaths from lung cancer increasingly outnumber other cancer deaths due to maturation of the tobacco epidemic in Asia and eastern Europe, and to declining death rates from cancers of the stomach, liver, cervix, and oesophagus in many developing countries. The cancers that have been common historically in developing countries are caused in part by infectious agents. Paradoxically, as these become less common, they are replaced by at least one cancer that is largely man-made.

Most deaths from lung cancer in other countries continue to be in men, although in the United States, women now contribute $47 \%$ of the 171500 new cases and $42 \%$ of the 160100 deaths expected to occur in $1998 .^{2}$ More women in the United States have died each year from lung cancer than from breast cancer since 1987. Although American women comprise only about $5 \%$ of women in the developed world, they accounted for more than $40 \%$ of lung cancer deaths attributable to smoking among women in developed countries in $1990 .^{3}$ Despite this extraordinary burden, lung cancer does not inspire the anxiety and public activism that are elicited by breast and prostate cancer. The most active constituencies working to reduce lung cancer are health agencies and the tobacco control community rather than the populations at highest risk.

\section{Mortality from lung cancer}

Improvements in therapy, mostly in surgical techniques, over the past 40 years have yielded better short-term survival from lung cancer. The one-year relative survival rate for all stages combined has been extended from 32\% in 1973 to $41 \%$ in 1994 ; however, the five-year relative survival rates for all stages remains at $14 \%{ }^{4}$ Only $15 \%$ of lung cancers are discovered when the disease is still localised and the five-year survival rate is $49 \%$. Recent, promising experimental studies show that anti-angiogenic drugs such as angiostatin and endostatin are effective in curing lung cancer in mice, yet this remains to be demonstrated in humans. 

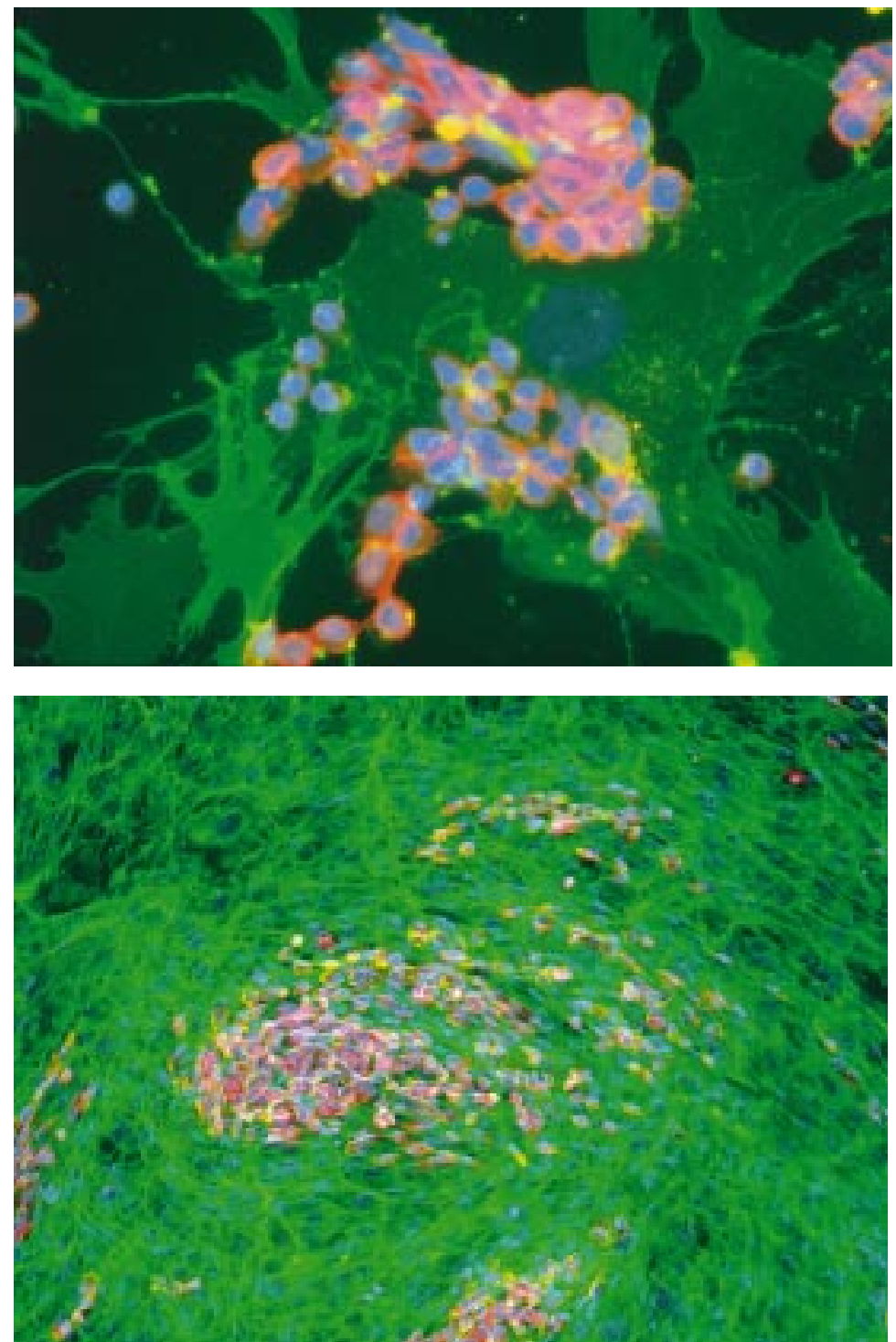

Immunofluorescent light micrograph of carcinoma cells of the lung. Carcinoma is the cancer that arises in epithelium, cells which line internal organs. Normal lung fibroblast cells are green in these images. Smaller, carcinoma cells (red) are seen growing upon this fibroblast lung tissue. Both cell types have blue nuclei. Immunofluorescence is a staining technique that uses antibodies to attach fluorescent dyes to specific tissues and to molecules within the cell. Magnifications: $\times 440$ (upper), $\times 230$ (lower). Source: Nancy Kedersha/Immunogen/ Science Photo Library (London). duration and intensity of smoking as well as on competing causes of death.

Three of the other known causes of lung cancer either derive from or interact with active smoking. Environmental tobacco smoke causes an estimated 3000 deaths from lung cancer among non-smokers annually in the United States. ${ }^{6}$ The risk of lung cancer from occupational exposure to radon or asbestos is amplified when either exposure is combined with tobacco smoke. Domestic radon exposure is estimated to cause 2100 to 2900 deaths annually among non-smokers in this country. Mendez et al have noted that reductions in smoking could effectively prevent many of the lung cancers caused by radon, because of the interaction between radon and tobacco smoke. ${ }^{8}$ Although occupational exposures to arsenic, cadmium, chromium, coke oven emissions, etc. have been reduced substantially in the United States, these remain important contributors to lung cancer risk in heavily exposed workers.

Smoking is believed to affect at least two stages of lung cancer development, based on clinical and statistical evidence. ${ }^{9}$ The genetic mutations that correspond to the early and late stages are less well defined for lung cancer than for colorectal cancer. It is known that the occurrence of early-stage event(s) in a population depends on the age at which people begin smoking regularly, the percentage who smoke, and how much they smoke. It is also known that the late stage, promoting effect(s) of smoking on lung cancer is dependent on continued smoking. Smokers who succeed in quitting, even in middle age, have a lower incidence of, and death rates from, lung cancer five or more years after cessation than do people of the same age who continue to smoke. ${ }^{10}$

The adverse impact of smoking on at least two stages of lung cancer development may explain a puzzling feature of the lung cancer epidemic among American men. Despite widespread smoking cessation among men in the late 1950 s and 1960 s, and despite the known reduction in lung cancer risk among former smokers $10-15$ years after quitting, ${ }^{10}$ lung cancer incidence and mortality rates in American men did not begin to decrease until after 1987 . The probable explanation is that men who reached adolescence after the second world war began smoking more heavily at a younger age than did smokers of the previous generation who began smoking after the first world war. ${ }^{9}{ }^{11}$ The residual impact of their early regular smoking put them at much higher risk of lung cancer than were smokers in previous birth cohorts. This high risk was only partly offset by their subsequent quitting.

An apt analogy to the consequences of smoking on lung cancer in the short and long term is the example of a train that may or may not crash in a particular setting. The risk of a crash is increased by acceleration over the 20-40 miles (32-64 km) already travelled, but decreased by more recent deceleration during the last $10-15$ miles $(16-24 \mathrm{~km})$ of braking. The prolonged, complex temporal relationship between smoking and lung cancer makes it 
particularly difficult for citizens and policy makers to react adequately to the hazard.

Nevertheless, incidence of, and death rates from, lung cancer have reached a plateau and have began to decrease among American men since approximately 1990, and the rate of increase has begun to slow among women. ${ }^{4}$ The slowing of the epidemic follows an interesting and characteristic sequence in which risk first begins to decline among younger men, aged 30-49 (beginning as early as the mid-1970s in the United States) and then progressively declines in older age groups among whom the absolute lung cancer rates are much higher. Doll and Peto have characterised this progression as "the tail wagging the dog". ' Small absolute reductions in lung cancer risk in younger men become much larger absolute reductions at older ages and are reflected by a decrease in age-standardised rates as the relevant birth cohorts age.

A similar pattern is seen internationally, although countries can be divided into a few in which lung cancer occurrence is now decreasing in men, and a larger number in which rates continue to increase in men and are already or will soon increase in women. Downward trends in lung cancer incidence and mortality in men are now seen in the United States, the United Kingdom, Australia, Canada, and Scandinavia; the decline in lung cancer risk is substantially more advanced in the United Kingdom than in other countries. In contrast, most of the countries in eastern Europe and the former Soviet Union continue to exhibit extremely high lung cancer rates in men, with the epidemic only beginning in women. Curiously, in much of the world, lung cancer death rates provide the only barometer of the evolving pandemic of deaths from tobacco, ${ }^{3}{ }^{12}$ despite the fact that lung cancer accounts for fewer than half of all smoking-related deaths in most Western countries.

\section{Controversies}

Several subplots of the story relating tobacco to lung cancer periodically receive widespread attention. One is whether African American men who smoke have a higher risk of developing lung cancer than do white male smokers. Supporting this idea is that African American men begin smoking at later ages and smoke fewer cigarettes per day on average, yet have higher incidence of, and death rates from, lung cancer than do white men. Reasons that have been proposed include the greater use of mentholated cigarettes by African American than by white men, more intense smoking (possibly aided by the anaesthetic effect of menthol), poorer nutrition, less access to health care that might lead to early diagnosis, and genetic factors. African American male smokers do have lower urinary clearance of cotinine ${ }^{13}$ and higher serum cotinine concentrations per cigarette than white or Mexican American smokers. ${ }^{14}$ Various genetic polymorphisms possibly associated with lung cancer risk are also more common among African Americans.

A second controversy is whether women are more susceptible than men to develop lung cancer from smoking. Higher relative risk estimates have been observed among women than among men in some case-control studies. This has not been supported by prospective studies, however, where generally the opposite is true; the absolute and relative risks of lung cancer death associated with smoking are greater among men than among women. The important point, however, is not whether men or women are subtly different in their susceptibility to smoking-related lung cancer, but that both sexes are highly susceptible if they smoke comparably.

\section{Other special issues}

At least four other issues deserve brief mention. First, based on the large prospective studies reported by the American Cancer Society, the lung cancer death rate approximately doubled among male current smokers and increased over five-fold among female current smokers from the early 1960 s to the mid-1980s, but remained essentially unchanged over the same interval among lifelong non-smokers. ${ }^{11}$ The epidemic of lung cancer observed in the general American population is largely confined to smokers.

Second, the histopathological distribution of lung cancer has changed over the past 40 years in North America, Europe, and parts of Asia such that adenocarcinoma, once considered to be minimally related to cigarette smoking, has become the most common cell type in the United States. ${ }^{15}$ In the American Cancer Society studies, adenocarcinoma has also become more strongly associated with cigarette smoking over time. Furthermore, the increase in adenocarcinoma in population-based registries such as the one in Connecticut follows a clear birth cohort pattern, more closely paralleling gender and generational changes in cigarette design (the introduction of filter-tip and lower yield cigarettes) than secular improvements in diagnosis.

Third, early progress has been made in identifying genetic markers of risk for developing lung cancer. These include genetic polymorphisms that code for certain cytochrome P450 enzymes such as CYP 1A1 MSP1 on chromosome 15 , rapid arylamine $\mathrm{N}$-acetyltransferase (NAT-2), and polymorphisms of glutathione S-transferase. It is not yet known to what extent these genes are clinically important, or whether the potential mechanism involves enhanced activation of carcinogens in tobacco smoke, reduced capacity to metabolise and thus detoxify carcinogens, or increased susceptibility to nicotine addiction. Moreover, no established screening methods exist to detect preclinical lung cancer, nor would a person not genetically susceptible to lung cancer be safe against the many other adverse effects of smoking.

Fourth, the Preventive Services Task Force in the United States currently does not recommend routine screening for lung cancer with chest radiography or sputum cytology in asymptomatic persons, but does recommend that all patients be counselled against tobacco use. The issue of screening is reconsidered 
periodically and could be affected by the increasing occurrence of peripheral lung tumours that might be diagnosed at an earlier stage when the cancer is still resectable.

The only form of chemoprevention recommended to reduce the risk of lung and other cancers is a diet high in fresh vegetables and fruit. ${ }^{16}$ Vitamin supplementation with beta-carotene is not recommended because of the finding in two of three randomised clinical trials of smokers that found increased lung cancer occurrence in smokers treated with beta-carotene.

Finally, do not expect to be thanked for your efforts to end the lung cancer epidemic. Teenagers will not be thankful, because threats of distant mortality seem merely irrelevant and annoying to them. Educated adults will not thank you, because few either still smoke or care about cancers they are unlikely to get. Smokers might appreciate a device that made it easier to quit. Perhaps our most enthusiastic audience will be elementary school and preschool children, who care about their lungs, and could learn to recognise and reject tobacco advertising. We will need their help to continue making progress against cancer.

1 Murray CJL, Lopez AD. Global health statistics, vol II. Cambridge, Massachusetts: Harvard University, 1996:183-4.

2 Landis WH, Murray T, Bolden S, et al. Cancer statistics 1998. CA Cancer f Clin 1998;48:31-48.

3 Peto R, Lopez AD, Boreham J, et al. Mortality from smoking in developed countries: 1950-2000. New York: Oxford in developed countries:

4 Ries LAG, Kosary CL, Hankey BR, et al, eds. SEER cancer statistics review, 1973-1995. Bethesda, Maryland: National Cancer Institute, 1998.
5 Johnston-Davis K, Wingo PA, Thun MJ, et al. Probabilities of lung cancer death associated with smoking: changes from the 1960 s to the 1980 s. Proceedings of the Meetings of the Society of Behavioral Medicine, 1997.

6 US Environmental Protection Agency. Respiratory health effects of passive smoking: lung cancer and other disorders. Smoking and Tobacco Control Monograph No 4. Bethesd Bethesda, Maryland: US Department of Health and tutes of Health, 1993. (NIH Publication No 93-3605.)

7 National Research Council, Committee on Health Effects of Exposure to Radon (BEIR), Commission on Life Sciences, et al. Health effects of exposure to radon. BEIR VI. Washington, DC: National Academy Press, 1998.

8 Mendez D, Warner KE, Courant PN. Effects of radon mitigation vs smoking cessation in reducing radon-related risk of lung cancer. Am f Public Health 1998;88:811-2.

9 Doll R, Peto R. The causes of cancer. F Natl Cancer Inst 1981;66:1191-308.

10 US Department of Health and Human Services. The health benefits of smoking cessation. A report of the Surgeon General, 1990. Rockville, Maryland: Public Health Service, Centers for Disease Control, Office on Smoking and Health, 1990. (DHHS Publication No (CDC) 90-8416.)

11 Thun MJ, Day-Lally C, Myers DG, et al. Trends in tobacco smoking and mortality from cigarette use in Cancer Prevention Studies I (1959 through 1965) and II (1982 through 1988). In: Changes in cigarette-related disease risks and their implication for prevention and control. Bethesda, Maryland: National Cancer Institute, 1997: chapter 4. (NCI Monograph No 8.)

12 Lopez AD, Collishaw NE, Piha T. A descriptive model of the cigarette epidemic in developed countries. Tobacco Control 1994;3:242-7.

13 Pérez-Stable EJ, Herrera B, Jacob P III, et al. Nicotine metabolism and intake in black and white smokers. $\mathcal{F} A M A$ 1998;280:152-6.

14 Caraballo RS, Giovino GA, Pechacek TF, et al. Racial and ethnic differences in serum cotinine levels of cigarette smokers. FAMA 1998;280:135-9.

15 Thun MJ, Lalla CA, Flannery JT, et al. Cigarette smoking and changes in the histopathology of lung cancer. $\mathcal{F}$ Natl Cancer Inst 1997;89:1580-6.

16 American Cancer Society 1996 Advisory Committee on Diet, Nutrition, and Cancer Prevention. Guidelines on diet, nutrition and cancer prevention: reducing the risk of cancer with health food choices and physical activity. $\mathrm{Ca}$ Cancer $\mathcal{F}$ Clin 1996;46:325-41.

\section{Note to readers}

We hereby solicit your ideas and contributions for future covers of Tobacco Control. As with previous covers, we would like future covers to be colourful and creative-with a tobacco control theme. Original artwork, anti-tobacco posters, photographs, and cartoons may all be considered. Material with an international flavour would be particularly desirable. A cover essay will generally appear in each issue to provide appropriate background information and commentary on the cover.

Please send ideas and submissions (original or high-quality, camera-ready photographs) to the editor at the address on the inside front cover.- $-\mathrm{ED}$ 